\title{
Structural Evolution in Photoactive Yellow Protein Studied by Femtosecond Stimulated Raman Spectroscopy
}

\author{
R. Nakamura ${ }^{1}$, N. Hamada ${ }^{1}$, K. Abe ${ }^{2}$, and M. Yoshizawa ${ }^{2}$ \\ ${ }^{1}$ Venture Business Laboratory, Osaka University, Suita 565-0871, Japan \\ ${ }^{2}$ Graduate School of Science, Tohoku University, Sendai 980-8578, Japan
}

\begin{abstract}
Ultrafast structural evolution in photoactive yellow protein (PYP) is studied by femtosecond stimulated Raman spectroscopy. A comparison between wild-type PYP and E46Q mutant reveals that the hydrogen-bonding network surrounding the chromophore of PYP is immediately rearranged in the electronic excited state.
\end{abstract}

\section{Introduction}

Photoactive yellow protein (PYP) is a 125-residue, $14 \mathrm{kDa}$ photoreceptor protein isolated from Ectothiorhodospira halophile. The chromophore of PYP is a 4-hydroxycinnamic acid which is covalently bound to Cys69 through a thioester linkage. In the ground state, the chromophore is in a deprotonated trans form stabilized through a hydrogen-bonding network with Glu46, Tyr42, and Cys69 (see Fig. 1). After photoexcitation, PYP undergoes a photocycle with a number of intermediate states $\left(\mathrm{I}_{0}, \mathrm{I}_{1}, \mathrm{I}_{2}\right)$, which involves trans-cis isomerization of the chromophore, rearrangement of the hydrogen-bonding network surrounding the chromophore, and large structural changes of the protein. PYP returns from the $\mathrm{I}_{2}$ state to its initial ground state in a few hundred milliseconds [1].

Ultrafast infrared spectroscopies have demonstrated that the trans-cis isomerization of the chromophore is completed in the $\mathrm{I}_{0}$ state of PYP within a few picoseconds [2,3], which is accompanied with dynamic changes of the hydrogen-bonding network surrounding the chromophore. For example, the hydrogen bond between the chromophore and Glu46 is weakened in the electronic excited state, and subsequently strengthened during the $\mathrm{I}_{0}$ state formation [2].

To investigate the hydrogen-bonding network participating in ultrafast photoinduced dynamics of the chromophore in PYP, we perform femtosecond stimulated Raman spectroscopy (FSRS) for wildtype (WT) and the E46Q mutant of PYP. In the E46Q mutant, the network is modified to have a weakened hydrogen bond from Gln46 to the chromophore phenolate oxygen. Recently, picosecond UV resonance Raman spectroscopy of Tyr42 in PYP have revealed that in the electronic excited state the structure of the hydrogen-bonding network around the chromophore of WT is similar to that of E46Q [4]. In this study, we reveal ultrafast dynamics of the chromophore and the surrounding hydrogen-bonding network in PYP with 150-fs time resolution.

\section{Experimental}

FSRS setup is based on an amplified mode-locked Ti:Sapphire laser system operating at $1 \mathrm{kHz}$ [5]. A fraction of the amplified pulses was used to drive two independent optical parametric 
amplifiers (OPA): the femtosecond OPA pumped by the fundamental pulses $(800 \mathrm{~nm})$ and the narrowband OPA pumped by the second harmonics $(400 \mathrm{~nm})$. The femtosecond OPA was used to generate the excitation pulse $(460 \mathrm{~nm}, 100 \mathrm{fs}, 80 \mathrm{~nJ})$, which was obtained by the four-wave summixing process $\left(\omega_{\mathrm{ex}}=\omega_{\mathrm{p}}+\omega_{\mathrm{i}}+\omega_{\mathrm{i}}\right)$ in a type I BBO crystal with the fundamental pulse $\left(\omega_{\mathrm{p}}\right)$ and the idler pulse $\left(\omega_{\mathrm{i}}\right)$. On the other hand, the narrowband OPA was used to generate the Raman excitation pulse $\left(520.5 \mathrm{~nm}, 32 \mathrm{~cm}^{-1}, 80 \mathrm{~nJ}\right)$. In the narrowband OPA, the seed pulse was spectrally filtered before and after amplification by using a spectral filter consisting of a pair of gratings and a slit. The femtosecond supercontinuum generated by a sapphire plate was used as a broadband probe pulse. The full width at half maximum (FWHM) of the cross correlation traces between the excitation and probe pulses was $150 \mathrm{fs}$.

WT and E46Q PYP were prepared as described previously [6]. The sample was dissolved in 100 $\mathrm{mM}$ Tris- $\mathrm{HCl}$ buffer $(\mathrm{pH} 7.5)$. The optical density of both samples is 1.0 per $\mathrm{mm}$ at the absorption maximum.

\section{Results and Discussion}

Figure 1(C) shows the stimulated Raman spectra on the Stokes side of the initial ground state of WT (solid line) and E46Q (broken line), which exhibit vibrational features related to coupled $\mathrm{C}=\mathrm{C}$ and $\mathrm{C}-\mathrm{C}$ stretching modes in the $1450-1600 \mathrm{~cm}^{-1}$ region, coupled $\mathrm{C}=\mathrm{C}$ (or $\mathrm{C}-\mathrm{C}$ ) stretching and inplane $\mathrm{CH}$ rocking modes in the $1100-1350 \mathrm{~cm}^{-1}$ region, and skeleton or out-of-plane vibrational modes in the region below than $1000 \mathrm{~cm}^{-1}$ [7]. The intense Raman band at $1555 \mathrm{~cm}^{-1}$, which contains both the vinyl bond $\mathrm{C}=\mathrm{C}$ stretching and the ring vibrations analogous to $\mathrm{Y} 19 \mathrm{a}$ of tyrosine, is known to be sensitive to the hydrogen bond of the phenolate oxygen of the chromophore. Remarkable difference of this Raman band for between WT and E46Q is known as a result of alternation by the E46Q mutation [8]. We focus on its relative intensity in this study.
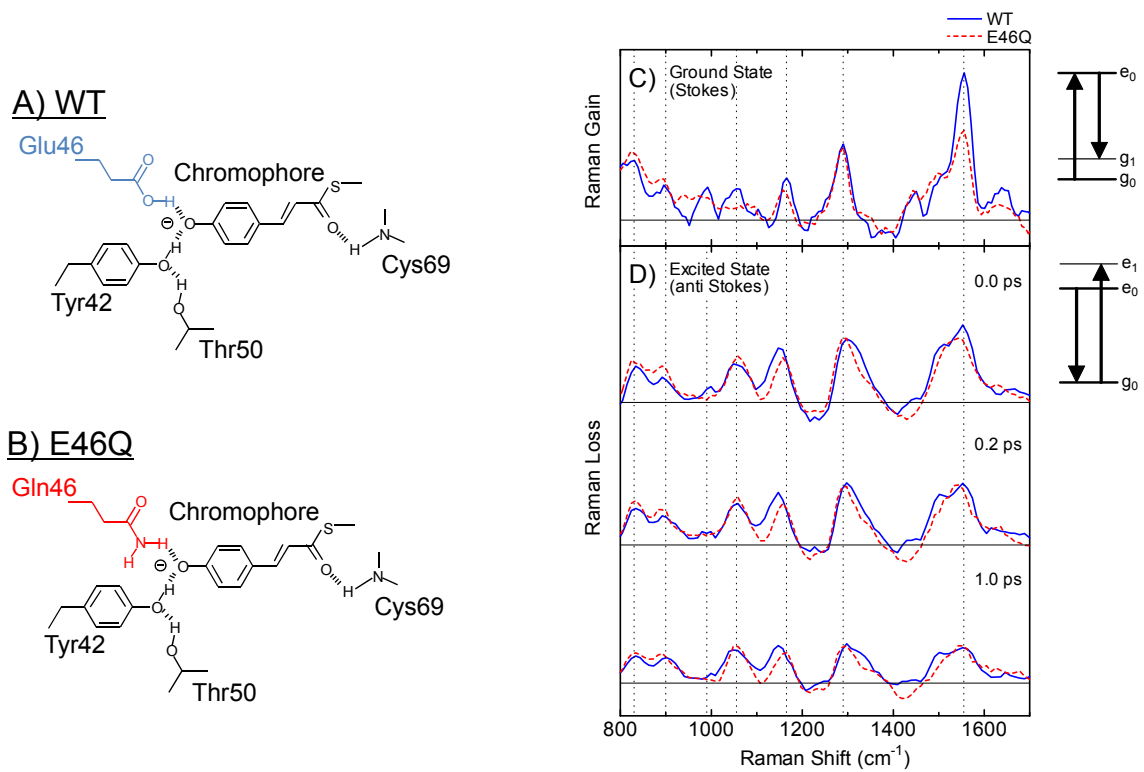

Fig. 1. Schematic depictions of the chromophore with its surrounding hydrogen-bonding network for (A)WT PYP and (B) E46Q mutant. (C) Stimulated Raman signals on the Stokes side of the initial ground state. (D) Femtosecond stimulated Raman signals on the anti-Stokes side measured at selected delay times after photoexcitation. Diagrams depicted at the right-hand side of the figure are the corresponding Raman processes, where $\mathrm{g}_{0}\left(\mathrm{~g}_{1}\right)$ and $\mathrm{e}_{0}\left(\mathrm{e}_{1}\right)$ are vibrational ground (excited) states belonging to the electronic ground and excited states, respectively. 
Figure 1(D) shows the transient stimulated Raman spectra on the anti-Stokes side at selected time delays after photoexcitation. The Raman excitation energy used in this study is much smaller than the transition energy from the first excited state $S_{1}$ to the higher excited state $S_{2}$. Instead, the Raman excitation is resonant to the transition from $S_{1}$ to the ground state $S_{0}$. We measured energy dependence of the Raman excitation pulse on the stimulated Raman spectra on both the Stokes and anti-Stokes sides, and compared them with numerical calculations based on the density matrix treatment. We then confirmed that the observed Raman signal on the anti-Stokes side was attributed to the Raman process with vibrational coherence in the electronic excited state as depicted in the right-hand side of Fig. 1(D) [9].

The relative intensity of the Raman band at $1555 \mathrm{~cm}^{-1}$ for WT is depleted in the electronic excited state compared with the ground state Raman spectrum, while that for E46Q does not change with photoexcitation. Consequently, the relative intensities of the Raman band in the electronic excited states of WT and E46Q become similar to each other as shown in Fig. 1(D). This result is consistent with the UV resonance Raman study revealing that the structure of the hydrogen-bonding network in the electronic excited state of WT becomes similar to that of E46Q [4]. Our observation provides the following picture: In the initial ground state of WT, the vibrational mode at $1555 \mathrm{~cm}^{-1}$ is strongly coupled to the electric transition of $\mathrm{S}_{0}-\mathrm{S}_{1}$. After photoexcitation, the hydrogen bond of the phenolate oxygen of the chromophore suddenly reforms into the similar structure to that of E46Q within our time resolution of $150 \mathrm{fs}$. This ultrafast reformation is consistent with ultrafast relaxation process from the Franck-Condon state observed with time-resolved fluorescence spectroscopy [10]. According to the previous reports [2,3], this reformation presumably corresponds to weakening of the hydrogen bond between Glu46 and the phenolate oxygen. For the chromophore with the hydrogen-bonding network structure at the equilibrium of the excited-state potential surface, the coupling of the vibrational mode at $1555 \mathrm{~cm}^{-1}$ to the $\mathrm{S}_{1}-\mathrm{S}_{0}$ transition decreases and then results in a decrease of the Raman intensity.

\section{Conclusion}

We studied structural evolution of the chromophore in photoactive yellow protein in the primary stage of the photocycle by femtosecond stimulated Raman spectroscopy. By comparing wild-type PYP with E46Q mutant, it was revealed that rearrangement of the hydrogen-bonding network surrounding the chromophore occurred in the electronic excited state.

\section{References}

1. K.J. Hellingwerf, J. Hendriks, and T. Gensch, J. Phys. Chem. A 107, 1082 (2003)

2. M.L. Groot, L.J.G.W. van Wilderen, D.S. Larsen, M.A. van der Horst, I.H.M. van Stokkum, K.J. Hellingwerf, and R. van Grondelle, Biochemistry 42, 10054 (2003)

3. K. Heyne, O.F. Mohammed, A. Usman, J. Dreyer, E.T.J. Nibbering, and M.A. Cusanovich, J. Am. Chem. Soc. 127, 18100 (2005)

4. M. Mizuno, H. Kamikubo, M. Kataoka, and Y. Mizutani, J. Phys. Chem. B 115, 9306 (2011)

5. M. Yoshizawa, R. Nakamura, O. Yoshimitsu, K. Abe, S. Sakai, K. Nakagawa, R. Fujii, M. Namgo, H. Hashimoto, Acta Biochim. Pol. 59, 49 (2012)

6. K. Mihara, O. Hisatomi, Y. Imamoto, M. Kataoka, and F. Tokunaga, J. Biochem. 121, 876 (1997)

7. M. Unno, M. Kumauchi, F. Tokunaga, and S. Yamauchi, J. Phys. Chem. B 111, 2719 (2007)

8. Y. Zhou, L. Ujj, T.E. Meyer, M.A. Cusanovich, and G.H. Atkinson, J. Phys. Chem. A 105, 5719 (2001)

9. R. Nakamura, N. Hamada, K. Abe, and M. Yoshizawa (to be submitted)

10. R. Nakamura, N. Hamada, H. Ichida, F. Tokunaga, Y. Kanematsu, J. Chem. Phys. 127, 215102 (2007) 